\title{
Compiler Directed Battery-Aware Implementation of Mobile Applications ${ }^{1}$
}

\author{
N. Z. Azeemi \\ Christian Doppler Laboratory for Design Methodology of Signal Processing Algorithms, \\ Institute of Communications and Radio Frequency Engineering, University of Technology Vienna, \\ Gusshausstrasse 25/389, A-1040 Vienna Austria \\ Emails: nzafar@nt.tuwien.ac.at
}

\begin{abstract}
Handheld embedded systems are crucial to obtain high performance for execution time as well as efficient battery usage. Unfortunately, most of the compilation techniques to obtain an efficient binary code for complex multimedia processors lack the optimal energy-cycle code. This work describes the methodology of the compilation for next generation handheld devices, which will support datacompute intensive applications at small form factors. We designed and implemented optimization framework which supports conventional code optimization scheme along with additional energy efficient benefits for multimedia applications such as MPEG-2 transcodec, H-264L transcodec. Optimization space is searched with genetic algorithm. Whole scheme reduces energy, on both as per cycle basis and as the total energy used over the lifetime of an application. The optimized G-728 audio codec meets realtime constraints on the Nexperia series of multimedia processor with low energy consumption. Furthermore, the performance improves by a factor of 0.489 and the energy consumption decreases by a factor of 0.203 over the baseline executable code.
\end{abstract}

\section{Keywords:}

Multi-Objective Optimization, Source Transformation, Battery-Aware, Multimedia Applications

\section{Introduction}

The tremendous increase in bringing multimedia functions on handheld devices posed a challenge to embedded application developers in term of many diversified goals, e.g., reduced development cost, low execution time, and optimal battery usage under hard real time constraints $[1,2,3]$. Reducing energy, on both as per cycle basis and as the total energy used over the lifetime of an application, has become more important as small and embedded devices become increasingly available. The later is implicitly connected to a demand for expensive packaging and cooling technology, an

\footnotetext{
${ }^{1}$ This work has been funded by the Christian Doppler Laboratory for Design Methodology of Signal Processing Algorithms.
}

increase in product cost, and a decrease in product reliability in all segments of the computing domains.

Traditional DSP compilers do not meet above mentioned efficient application goals [4]. In fact, the sophistication in current compilation techniques can be characterized as 'manufacturing implementation' of software, more or less like design and implementation for embedded hardware boards in their early development stage $[4,5]$. Though compilers have employed computation and data reordering to improve locality, this still requires expert analysis due to the obscured parallelism and communication patterns in traditional languages. Inline with this problem is ambiguity between the informal design methods and manual coding techniques. Software applications are developed with a partial or completely unawareness to the underlying hardware architecture.

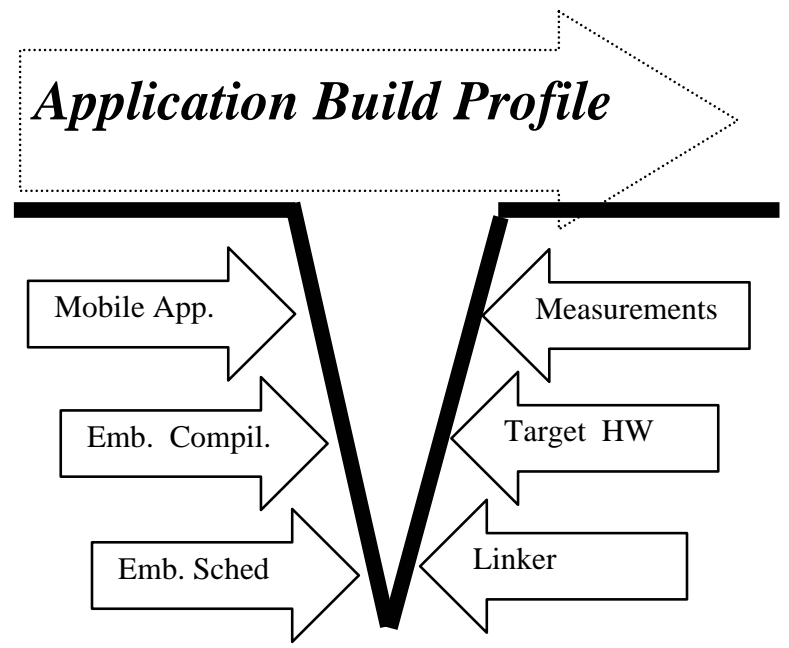

Figure 1. The ' $\mathrm{V}$ ' of Application Build Profile Monitors.

The crux of problem that has to be addressed in this context is to consider the application static and dynamic profile at all stages of application to binary build profile $[6,9]$. A framework can be defined as a contiguous 
executable models relevant to system architectural aspects and behavior within a given application domain. Embedded systems are inherently component, which interacts, process and port the data, which are later mapped onto real time systems.

We achieve this objective to some extent using vertical profiling approach and profile application at layers as shown in Figure 1. Multilevel profiling leads to a huge search space for an optimal objective solution [8]. An optimal solution seeking for each subtask is known to be an NP-hard optimization problem.

This paper approximates the NP-hard solution with the help of genetic algorithm. A genetic algorithm belongs to the class of stochastic optimization methods [26]. Although it does not guarantee finding of the global optimal solution, the result is a good approximation of it. Different optimization schemes are arranged based on application static profile and eventually fitness function is defined for a good solution.

The key contribution of this paper is that it demonstrates that significant potential exists for energy savings in multimedia applications without an undue increase in execution time. Therefore, it shows that code restructuring during iterative compilation is feasible and advantageous.

This paper is organized as follows: First we approach the problem of profiling from optimization viewpoint, and then we apply code restructuring algorithms in the context of energy-cycle optimization.

\section{Formalizing Platform-Based Framework}

Software application can express at different level of abstraction, starting from plain ' $c$ ' code until the binary generation and eventually execution at real time platform. Sections 2.1 describe our profile monitoring methodology. In Section 2.2 we formally define our objective function.

\subsection{Methodology Flow and Performance Monitors}

An embedded system can be viewed as a finite set of state machines, where a data dominated operations triggers the state transfer. E.g., control transfer with ifelse conditions. Such transfer may lead to cache misses. Therefore a careful consideration of state transfer event is necessary. In this work extend the state transfer monitors [7], here we call as performance monitors we described the methodology flow in our framework [7]. The VLIW ' $C$ ' source code is processed to extract profile monitoring at different layers of the framework (shown in Figure 2.).

An application is divided in to functional sub blocks and further into to atomic blocks. Each atomic block is independent of each other and performs only load/ store, logical/ arithmetical or transfer operations only. These transfer operations does not involve any conditional or logical operations. Conditional/ nonconditional branches are considered as one functional block. Following the static code analysis, E-C profiling is done at two layers of code, first, it is estimated for basic code blocks, each basic block has the same characteristics as mentioned above, followed by this, all sub blocks are assembled together to restore their functional level appearance and estimate is made again. Second, inter procedural effect is considered to cope up with the implicit cache miss, that leads to off-chip traffic and hence increases CPU cycles that inevitably leads to more energy consumption.

A pre/ post compiler analysis is scheduled later to optimize VLIW architectural usage. Architectural description is provided as a generic text file following a predefine header that consists of CPU, memory and onchip cache signatures. E.g., a list of pseudo and machine operations, the latency of the operations, the op codes, the slot assignment schemes, the processor operating frequency, the instruction cache feature as well as main memory features.

For the optimization space search we use genetic algorithm (GA). The application profile monitor capture session can be predefined in user constraints as endless and stopped manually. Additionally, the framework is equipped with an automatic optimization termination which adjusts the GA search space suitable to the desired objectives.

Current is measured at the target platform (the setup is explained in [7]. All these parameters are fed back to the transformation cost analyzer. In each successive transformation it is decided that whether energy-cycle performance has been optimized or not. Source code is optimized by undergoing code restructuring schemes known as loop unrolling, decision tree grafting, and loop tiling.

\subsection{Objective Function}

For every point lying on Pareto front in transformation space, we considered to optimize both CPU cycles for code execution and the energy consumption per process. To meet such objective it is necessary that the successive architecture utilization (in terms of functional units, internal register usage, best cache fit) must be greater than a predefined, system dependent 
limit (execution cycle and energy threshold). In the same vein, the predecessor transformation scheme must overlap the successor in order to follow a smooth optimization. The smooth optimization over two samples of code is defined by minimum and maximum limits of transformed code. If the output profile of code is between these limits, this point must lie on smooth curve for optimization.

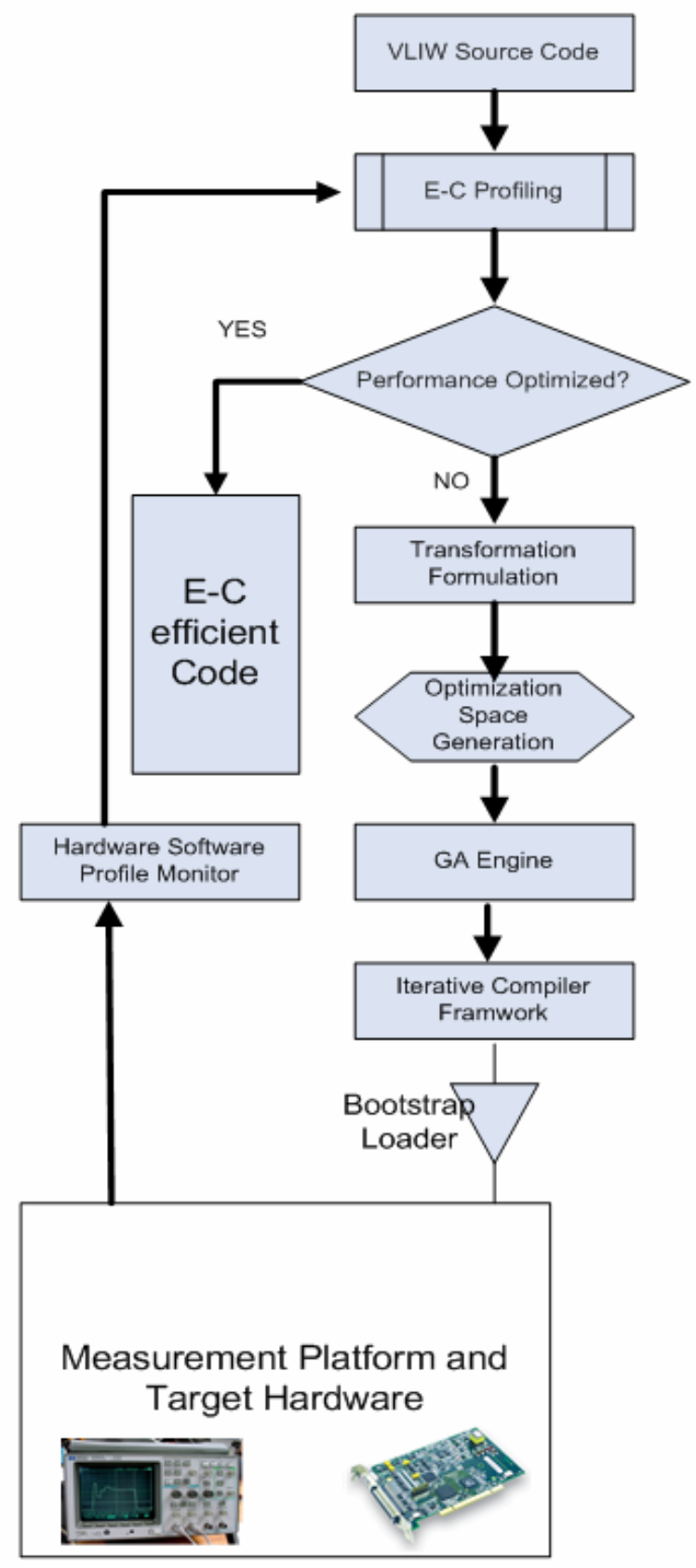

Figure 2. The Energy-Cycle Optimization Engine and Measurement Platform Framework.

\subsection{Fitness Function and Optimization Methodology}

The desired energy-cycle competition is optimized by introducing objective function as a single task and all constraints are modeled as linearly dependent on each other. This is a valid assumption, because our results [6] show that architectural utilization, energy consumption and CPU cycle efficiency can be approximated in linear relations. The desired aims of architecture-based energy-cycle optimization are formulated as penalty terms of such objective function. The maximization of objective function is achieved using a genetic algorithm (GA) [6, 12].

\section{Experimental Methods}

The new method of application profile monitoring has been investigated from different architectural attributes to show the variability of application and hence optimization potential for architecture efficient code generation.

\subsection{Benchmarks}

Multimedia applications use DSP algorithms and streaming data schemes to compute and later to produce high throughput for real time video or audio applications. The quality of throughput depends on the application domain, e.g., bandwidth and frame rate for a typical MPEG-2 application is different at mobile device and set-top box. We chose the application sfor their importance in real systems and to be representative enough to make the inferences in this study. This application set contains MPEG-1 transcodec, MPEG-2 transcode, G-728 transcodec and generic DSP algorithms ( iir, fir, dct, idct etc..). We obtained codes for these applications form various public domains sources. For lack of space, we only report their names, details may be found on public websites.

\section{$4 \quad$ Results and Discussion}

The main focus of this research is the optimization of multimedia application to an efficient energy cycle responses on hand-held or mobile devices. Therefore, binary code for different multimedia application domain was executed at the same target platform [13]. Both energy consumption and cycle efficiency were successfully measured to optimal architecture utilization. For these performance indices however a major drawback is the high architectural dependency. For the brevity, here we show optimization results for speech codec G-728enc, a high bit rate speech coding schemes based on G.728 standard. It used low delay 
CELP (code excited linearly prediction) coding at $16 \mathrm{Kbps}$, compress frame of five 16-bit samples into 10 bits). The code is inherently dominated with many branches between its different blocks, this feature restrain its effective utilization of parallelism offered by underlying hardware architecture. Figure 4.1 to Figure 4.4 show, code optimization for different optimization session during the framework flow. The highly branch oriented code limits multiple function unit utilization to only 60 percent, while cycle efficiency is improved to 50\% in Figure 4.1.

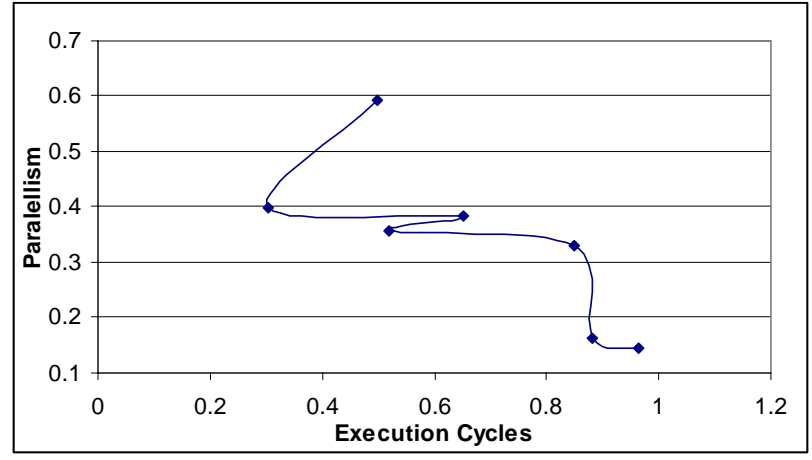

Figure 4.1. CPU Execution Cycles Correlation with Processor Parallelism.

The low cache hit in the beginning leads to higher number in execution cycle. An efficient search for appropriate loop fusion improves the code and hence results in better cache hits; leads to lower in execution cycles in Figure 4.2. At first glance, though cache hits is improved by $78 \%$, while cycle reduction was $25 \%$; but if you see the same result in conjunction with Figure 4.1, a different scenario will emerge. At $25 \%$ cycle reduction the benefits offered by architectural parallelism are only utilized by $40 \%$, while as we said earlier there is $20 \%$ more parallelism exists in architecture, which G.728enc can exploit. Similar conclusion can be made based on Figure 4.3, which show scheduling factor versus execution cycles.

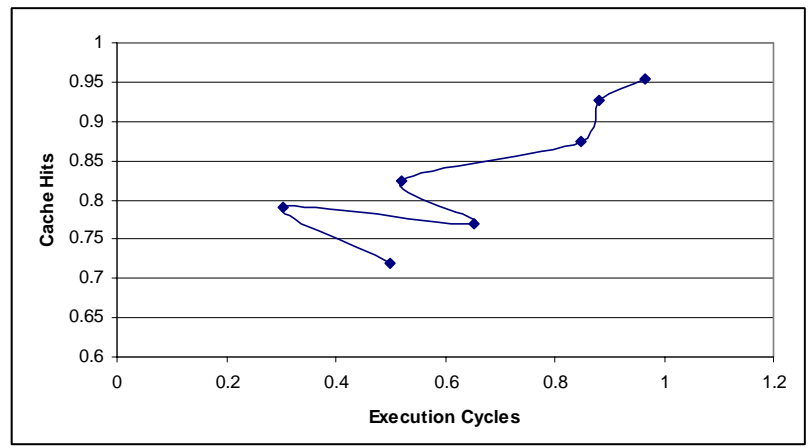

Figure 4.2. CPU Execution Cycles Correlation with Processor Cache Hits.
We obtain these results with the help of GA, and this is one salient feature that it does not stuck in local minima and always approximates a good solution rather than optimal solution based on multiple constraints.

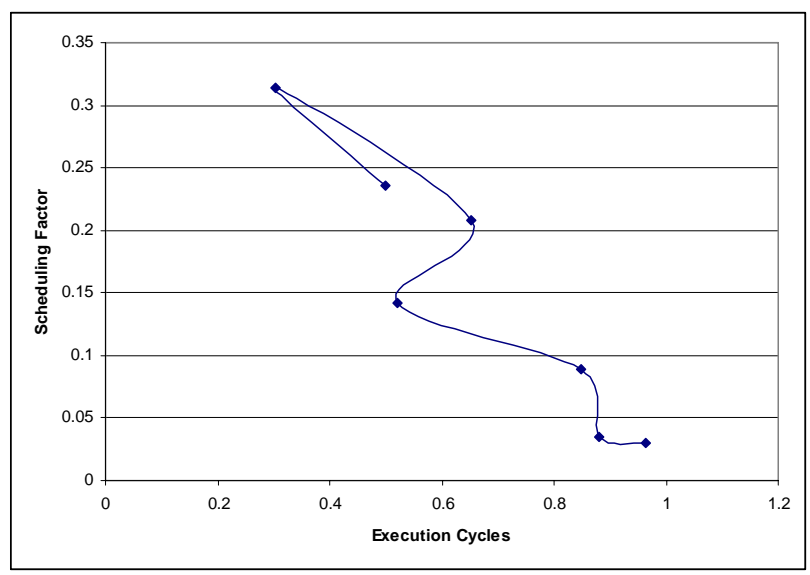

Figure 4.3. CPU Execution Cycles Correlation with Scheduling Factor.

Our target architecture offers highly parallelism [8], Figure 4.2 and Figure 4.4 show a potential dependence of energy saving at parallelism and cache hits. Trend of measured data show a linear correlation between the two. The steady state between the two energy knees in Figure 4.4 reveals an interesting fact, that not all applications can exploit a stat of the art parallelism for effective energy utilization. We may conclude that migration of application from one DSP to another DSP in mobile systems is not always promising.

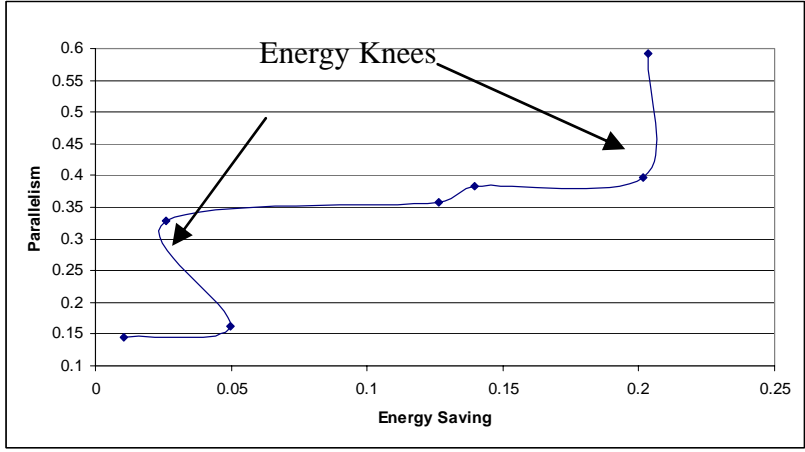

Figure 4.4. Energy Saving Correlation with Processor Parallelism.

Figure 4.5, depicts energy-cycle surface that reflect constrained driven convergence of fitness function in the direction of high energy saving and low CPU cycle execution (from right to left). S01 to S07 are awarded chromosome evolution session during GA search. The peaks in figure also show the impact of parametric 
optimization (both in term of architecture such as functional unit, cache misses, scheduling factor and the code static profile).

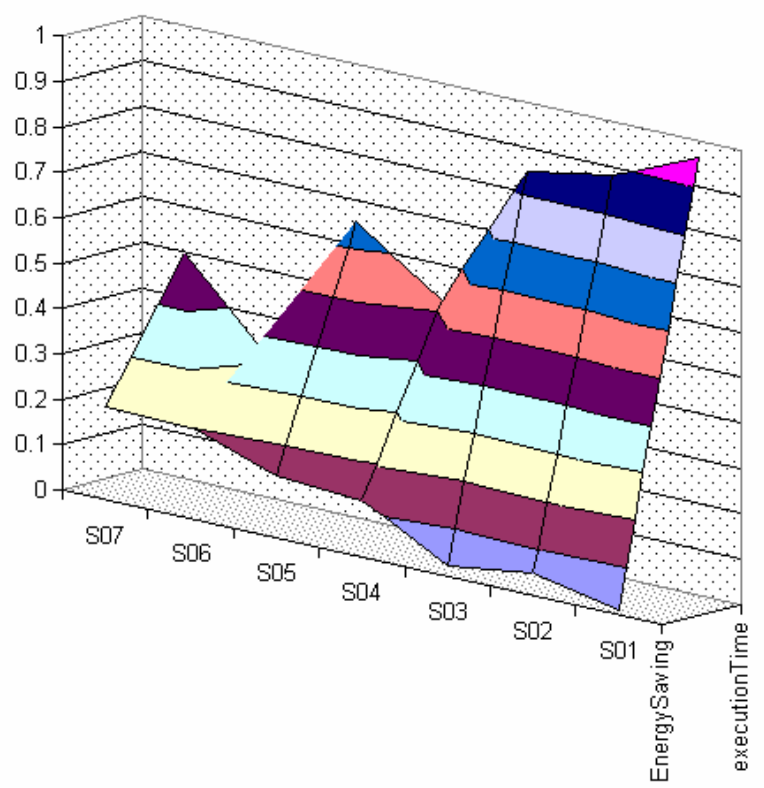

Figure 4.5. Transformation Contour for Multicritiria Optimization of G-728enc.

\section{Concluding Remarks}

In this paper, we address how by employing various techniques at the compiler level multimedia application can be optimized for a given hardware architecture. We demonstrate that it is necessary to profile application expression at all layers to understand existing performance problems such as poor architecture usage, increase in execution time, and high energy consumption. In particular presented how GA can be used to converge to energy-cycle efficient code generation. We demonstrate the proposed framework at commercial multimedia application with a significant performance gain. In case of G-728 speech codec, by carefully restructuring code the and the control flow in source code energy saving as much as $20 \%$ and cycle efficiency upto 50\% can be achieved. More work needs to be done to accurately estimate the energy cost of software including operating systems, API interfaces and applications written in embedded $\mathrm{C}++$ or embedded JAVA.

\section{Acknowledgment}

This work is supported by ÖAD-Pakistan scholarship program initiated by Prof. Dr. Atta-ur-Rahman chairman HEC and Federal Minister Pakistan. We thankful to Prof. Dr. Markus Rupp, Prof. Dr. Arpad Scholtz and Christian Doppler Laboratory at Institute of Communication and Radio-Frequency Engineering, Vienna University of Technology for their support and kind input during this work.

\section{References}

[1] M.Lee, V.Tiwari, S.Malik, M.Fujita, '’Power Analysis and Minimization Techniques for Embedded DSP Software,', Proc. of the IEEE Trans on VLSI Design, pp.123-135, March 1997.

[2] C. Gebotys, R. Gebotys, S. Wiratunga, "Power minimization derived from architectural-usage of VLIW processors," Proc. of the Annual ACM IEEE Design Automation Conference, pp. 308-311, June 2000.

[3] C. Gebotys, R.Gebotys, .”Statistically based prediction of power dissipation for complex embedded DSP processors,” Micro-processors and Microsystems Journal, vol. 23, pp. 135-144, 1999.

[4] G. Fursin, M. O’Boyle, P. Knijnenburg, "Evaluating iterative compilation," Proc. of Languages and Compilers for Parallel Computers (LCPC’02), College Park, MD, USA, 2002.

[5] V. Tiwari, S. Malik, A. Wolfe, "Compilation techniques for low energy,” Proc. of the ISLPED, Oct 1994.

[6] N. Z. Azeemi, M. Rupp, “Muticriteria Low Energy Source Level Optimization of Embedded Programs,” Proc. of the IEEE Informationstagung Mikroelektronik 2006, pp. 150-158, Oct. 2006.

[7] N. Zafar, M. Rupp, "Energy-aware source-tosource transformations for a VLIW DSP processor," Proc. of the IEEE $17^{\text {th }}$ ICM 2005, pp. 133-138, Dec. 2005.

[8] N. Zafar Azeemi, "Power Aware Framework for Dense Matrix Operations in Multimedia Processors," Proc. of the IEEE 9th International Multi-topic Conference, Dec. 2005.

[9] Parameswaran, S. "Code placement in hardware/software co-synthesis to improve performance and reduce cost," Proc. of the Conference on Design, Automation and Test., pp 626-632, 2001.

[10] S. Bashford and R. Leupers, "Constraint driven Code Selection for Fixed-Point DSPs," Proc. of the 36th Design Automation Conference (DAC), Nov. 1999.

[11] T. Baeck. Evolutionary Algorithms in Theory and Practice. Oxford University Press, 1996 
[12] N. Zafar Azeemi, "A Framework for Architecture Based Energy-Aware Code Transformations in VLIW Processors," Proc. Of the International Symposium on Telecommunications (IST 2005) pp.393-398. Sep. 2005.

[13] TM1300 Data Book, Philips Electronic, North America Corporation, pp. 3.1-3.16, Oct 1999. 